\title{
Higgs Boson Production via Gluon Fusion in the Standard Model with four Generations
}

\author{
Qiang Li, ${ }^{1}$ Michael Spira, ${ }^{1}$ Jun Gao, ${ }^{2}$ and Chong Sheng $\mathrm{Li}^{2,3}$ \\ ${ }^{1}$ Paul Scherrer Institut, CH5232 Villigen PSI, Switzerland \\ ${ }^{2}$ Department of Physics and State Key Laboratory of Nuclear Physics and Technology, Peking University, Beijing, 100871, China \\ ${ }^{3}$ Center for High Energy Physics, Peking University, Beijing, 100871, China
}

(Received 22 November 2010; published 13 May 2011)

\begin{abstract}
Higgs bosons can be produced copiously at the LHC via gluon fusion induced by top and bottom quark loops, and can be enhanced strongly if extra heavy quarks exist. We present results for Higgs + zero-, oneand two-jet production at the LHC operating at 7 and $14 \mathrm{TeV}$ collision energy, in both the standard model and the 4th generation model, by evaluating the corresponding heavy quark triangle, box, and pentagon Feynman diagrams. We compare the results by using the effective Higgs-gluon interactions in the limit of heavy quarks with the cross sections including the full mass dependences. NLO effects on Higgs + zero-jet production rate with full mass dependence are presented for the first time consistently in the 4th generation model. Our results improve the theoretical basis for fourth generation effects on the Higgs boson search at the LHC.
\end{abstract}

DOI: 10.1103/PhysRevD.83.094018

PACS numbers: 14.65.Jk, 12.38.Bx, 14.80.Bn

\section{INTRODUCTION}

The Large Hadron Collider (LHC) is presently running with a c.m. energy of $7 \mathrm{TeV}$. One of its main goals is to explore the details of electroweak symmetry breaking, in particular, the Higgs sector of the standard model (SM). At the LHC Higgs boson production via gluon fusion, $g g \rightarrow H[1-3]$ is the dominant production mode. More exclusive channels such as Higgs associated production with one or two hard jets via gluon fusion or weak boson fusion [3,4,4-13] have also been studied. By using the accompanying jet information, one can refine the experimental cuts to increase the signal to background ratio and the potential of extracting Higgs parameters.

The measurement of Higgs boson production at the LHC can also give hints or constraints on those new physics beyond the SM which couple to the Higgs sector, such as e.g. the fourth generation extension of the SM (SM4) (see, for example, [14-18]), which assumes additional heavy quarks $\left(b^{\prime}, t^{\prime}\right)$ with Higgs Yukawa interactions growing with their masses as for the SM quarks. The SM4 can address some of the current open questions, e.g. it provides new sources for $C P$ violation [19] and baryogenesis $[20,21]$. The SM4 is also consistent with the precision electroweak data. In particular, the SM4 fit can lead to a much higher upper limit on the Higgs mass of $\sim 750 \mathrm{GeV}$ at $95 \%$ C.L. $[15,16]$ than the SM one, and thus reduces the tension with the $114.4 \mathrm{GeV}$ lower limit from LEP2 [22,23]. Recently, the Tevatron constrained the 4th-generation $b^{\prime}$ mass to $m_{b^{\prime}}>338 \mathrm{GeV}$ [24], while unitarity requirements bound the 4th generation $t^{\prime}$ mass as $m_{t^{\prime}}<504 \mathrm{GeV}$ $[18,25]$. Notice also that a SM4 Higgs with mass between $131 \mathrm{GeV}$ and $204 \mathrm{GeV}$ has been excluded at $95 \%$ C.L. by the Tevatron [26].

In the SM4, the appearance of heavy flavors enlarges the Higgs coupling to gluons significantly and thus the Higgs boson production rate at hadron colliders. Refs. [27,28] present the NNLO QCD results for Higgs inclusive production with effective Higgs-gluon interactions, and further incorporates the contributions of the top and extra heavy quarks consistently following the method of Ref. [29]. In Ref. [16], Higgs + 2-jet production via gluon fusion has been studied as a background to the weakboson-fusion process by using the effective Higgs coupling to gluons. In this paper, we investigate Higgs + jet(s) associated production, and check, in particular, the validity of the approach of using the effective Higgs-gluon interactions in our case. This paper is organized as follows. In Sec. II, we describe the calculation. In Sec. III, we present numerical results and their discussion. Finally, we conclude in Sec. IV.

\section{DESCRIPTION OF THE CALCULATION}

At the LHC, Higgs +1 -jet and 2-jet production via gluon fusion receives contributions from the partonic processes

$$
g g \rightarrow g h, \quad g q \rightarrow q h, \quad q \bar{q} \rightarrow g h,
$$

and

$$
\begin{gathered}
g g \rightarrow g g h, q \bar{q} h, \quad g q \rightarrow g q h, \quad q q \rightarrow q q h, \\
q q^{\prime} \rightarrow q q^{\prime} h, \quad q \bar{q} \rightarrow g g h, q \bar{q} h, q^{\prime} \bar{q}^{\prime} h,
\end{gathered}
$$

respectively, where $q^{\prime}$ denotes quarks with different flavor than $q$.

The relevant one-loop Feynman diagrams and amplitudes for all the subprocesses mentioned above have been generated with FeynArts 3.5 [30], and manipulated with FormCalc 5.3 [31]. The Fortran libraries generated with FormCalc are then linked with our Monte Carlo integration code for final use, in which we have modified the codes generated with FormCalc by adding the extra heavy flavor 
contributions of the SM4. The tensor integrals are evaluated with the help of the LoopTools-2.5 package [31], which employs the reduction method introduced in Ref. [32] for pentagon tensor, and Passarino-Veltman reduction for the lower point ones. The resulting regular scalar integrals are evaluated with the FF package [33]. Note that the uv and ir divergent scalar integrals have already been encoded into this newest version of LoopTools within dimensional regularization, which we have checked with QCDloop [34]. Moreover, we have modified LoopTools-2.5 to implement the reduction method for pentagon tensor integrals as proposed in Ref. [35], and found it leads to much better numerical stability in our case.

We have performed a second calculation based on the heavy-top effective Lagrangian [2,36], which is a good approximation for not too heavy Higgs bosons $\left(M_{h} \lesssim\right.$ $\left.m_{t}\right)$ and in appropriate kinematic regions [8]:

$$
\mathcal{L}_{\text {eff }}=n_{h} \frac{\alpha_{s}}{12 \pi v} H G_{\mu \nu}^{a} G^{a \mu \nu},
$$

where $G_{\mu \nu}^{a}$ denotes the gluon field strength tensor, and $n_{h}$ represents the number of heavy quarks, i.e. $n_{h}=1$ (3) for the SM (SM4). This effective model has already been implemented in MadGraph4 [37], with which we generated all contributing tree level Feynman diagrams and helicity amplitudes for the processes listed in Eqs. (2.1) and (2.2). The numerical evaluation is then performed by using the HELAS library [38].

We have checked our calculations in several ways. First, we compared the results of our two calculations and got good agreement between them for smaller Higgs masses as expected. Second, we compared the SM result with Ref. [8] and could reproduce their results with the same settings and parameter choices.

\section{NUMERICAL RESULTS}

In this section, we present the total cross sections and differential distributions for Higgs +1 -jet and 2-jet production at the LHC. For completeness, we also show the relevant Higgs +0 -jet results at LO and NLO.

We impose the minimal set of cuts

$$
\left|\eta_{j}\right|<4.5, \quad P_{T}^{j}>P_{T}^{\text {cut }}, \quad \Delta R_{j j}=\sqrt{\Delta \eta^{2}+\Delta \phi^{2}}>0.6
$$

to identify massless partons with jets. Here, $\eta$ is the pseudorapidity of the jets and $\phi$ is the azimuthal angle around the beam direction. $P_{T}^{\text {cut }}$ is the jet transverse momentum cut, chosen as a function of the Higgs mass $M_{h}$ :

$$
P_{T}^{\text {cut }}=0.04 M_{h}+14 \mathrm{GeV}
$$

thus, for example, $P_{T}^{\text {cut }}=30 \mathrm{GeV}$ for $M_{h}=400 \mathrm{GeV}$, which ensures the perturbative reliability of our results over a wide range of Higgs mass, i.e. $\sigma_{2 j}<\sigma_{1 j}<\sigma_{0 j}$.
We also show the results for Higgs +2 -jet production with the following weak-boson-fusion cuts in addition:

$\left|\eta_{j 1}-\eta_{j 2}\right|>4, \quad \eta_{j 1} \cdot \eta_{j 2}<0, \quad m_{j j}>600 \mathrm{GeV}$.

Throughout our calculation, we set the top quark mass to $m_{t}=173.0 \mathrm{GeV}$ and take the five flavor scheme to treat external bottom quarks as massless particles, while keeping the bottom quark mass as $m_{b}=4.6 \mathrm{GeV}$ in the fermion loops. The Fermi constant has been chosen as $G_{F}=1.16637 \times 10^{-5} \mathrm{GeV}^{-2}$. For the LO results, we adopt the CTEQ6L1 parton distribution functions (PDFs) [39] with the corresponding value for the LO strong coupling $\alpha_{s}\left(M_{Z}\right)=0.130$, while at NLO we used the CTEQ6.6M PDFs with the NLO strong coupling normalized to $\alpha_{s}\left(M_{Z}\right)=0.118$. Our default choice for the renormalization and factorization scales is $M_{h}$.

For the SM4 parameters, we first focus on the scenario as chosen in Ref. [28]:

$$
m_{b^{\prime}}=400 \mathrm{GeV}
$$

and

$$
m_{t^{\prime}}=m_{b^{\prime}}+50 \mathrm{GeV}+10 \log \left(\frac{M_{h}}{115 \mathrm{GeV}}\right) \mathrm{GeV},
$$

which is consistent with electroweak precision tests [16]. We will also discuss the dependence on $m_{b^{\prime}}$.

Figure 1 shows the Higgs $+n-$ jet $(n=0,1,2)$ production cross sections as functions of the Higgs boson mass at the LHC with $\sqrt{s}=7$ and $14 \mathrm{TeV}$, including the minimal cuts of Eq. (3.1). As expected, in the light Higgs boson region $\left(M_{h} \lesssim 200 \mathrm{GeV}\right)$ the gluon-fusion results agree well with the ones obtained by using the effective Higgs coupling to gluons for both the SM and SM4. The visible discrepancy for the $n=0$ case in the light Higgs boson region is due to the contribution of the bottom quark loops at the $10 \%$-level, which is much smaller for Higgs +1 -jet and 2-jet production. For light Higgs bosons, one can approximate the enhancement ratio of the SM4 rate over the $\mathrm{SM}$ one as $n_{h}^{2} \approx 9$. In the larger Higgs mass region, the effective Higgs coupling approximation breaks down, and the gluon-fusion results differ significantly from the effective Higgs coupling approximation, especially when $M_{h}$ is near the thresholds at $M_{h} \sim 2 m_{t, b^{\prime}, t^{\prime}}$, where threshold effects play a role. For heavy Higgs bosons with $M_{h} \gtrsim 800 \mathrm{GeV}$ the Higgs +1 -jet and 2-jet production cross sections at the LHC with $\sqrt{s}=14$ (7) $\mathrm{eV}$ in the SM4 can exceed the SM one by more than a factor of 10 and amount to $\mathcal{O}(100) \mathrm{fb}(\mathcal{O}(10) \mathrm{fb})$, which is promising for the related Higgs boson searches at the LHC.

In Fig. 2, we show the cross sections as functions of $M_{h}$ for Higgs +2 -jet production via gluon fusion in both the SM and SM4, and also display the SM results via the weakboson-fusion process, imposing both the minimal and weak-boson-fusion cuts of Eqs. (3.1) and (3.3). Again, 

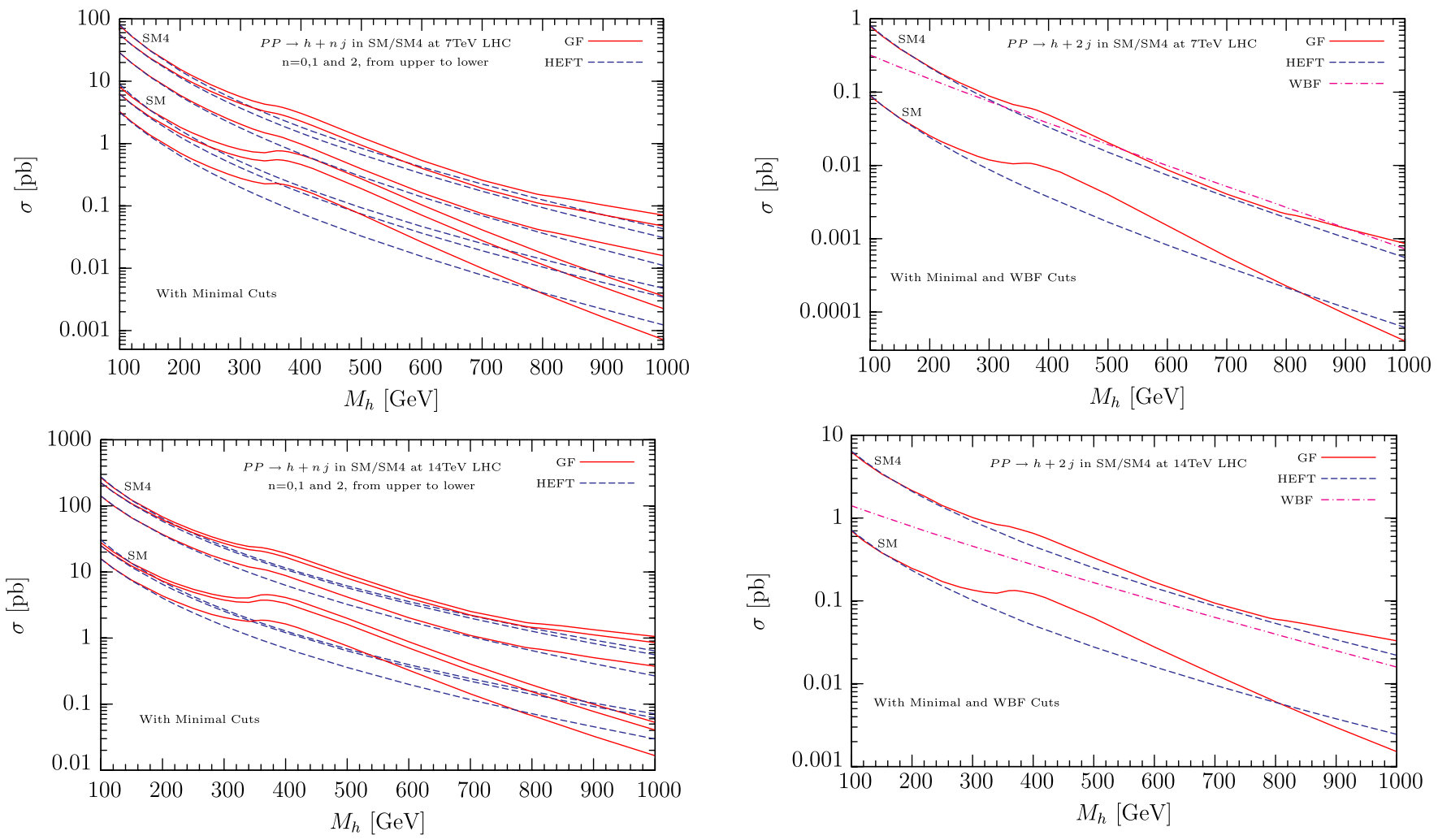

FIG. 1 (color online). Cross sections of Higgs $+n$-jet $(n=0$, $1,2)$ production via gluon fusion $(\mathrm{GF})$ in the SM and SM4 $\left(m_{b^{\prime}}=400 \mathrm{GeV}\right)$ at the LHC with $\sqrt{s}=7$ and $14 \mathrm{TeV}$ as functions of the Higgs boson mass with the minimal cuts of Eq. (3.1). The corresponding effective Higgs couplings results (HEFT) are also shown.

the effective Higgs coupling approximation works well for Higgs masses below about $200 \mathrm{GeV}$, for both the SM and SM4, even after imposing the large jet invariant mass cut in Eqs. (3.3), in agreement with Ref. [9]. One observes that the SM4 results are much larger than the SM ones, and can exceed the weak-boson-fusion results to a large extent, especially at the $14 \mathrm{TeV}$ LHC. Thus, the importance of the weak-boson-fusion channels will be diminished and the Higgs search strategies will be affected within the SM4 context.

Figure 3 displays the transverse momentum distribution of the harder jet for Higgs +2 -jet production via gluon fusion in the SM and SM4 at the $14 \mathrm{TeV}$ LHC, including the minimal cuts of Eq. (3.1) for $M_{h}=125 \mathrm{GeV}$ and $400 \mathrm{GeV}$, respectively. For both the SM and SM4, the effective Higgs coupling approximation works well if the Higgs mass and the $P_{T}$ of the harder jet is small $\left(P_{T} \lesssim m_{t}\right)$, as found in Ref. [9]. The agreement is better in the SM4 because the contributions of the heavier extra quarks dominate and thus postpone the breakdown of the approximation to larger Higgs boson mass values. However, the transverse momentum distribution of the harder jet is softer than in the effective Higgs coupling approximation in general.

FIG. 2 (color online). Cross sections of Higgs +2 -jet production via gluon fusion (GF) in the SM and SM4 $\left(m_{b^{\prime}}=400 \mathrm{GeV}\right)$ and via weak boson fusion (WBF) in the SM at the LHC with $\sqrt{s}=7$ and $14 \mathrm{TeV}$ as functions of the Higgs boson mass with both the minimal and weak-boson-fusion cuts of Eqs. (3.1) and (3.3). The corresponding effective Higgs coupling results (HEFT) for the gluon-fusion processes are also shown.

In Fig. 4, we show the azimuthal angle distribution between the two jets for Higgs +2 -jet production via gluon fusion in both the SM and SM4 $\left(m_{b^{\prime}}=400 \mathrm{GeV}\right)$ for $M_{h}=125 \mathrm{GeV}$ and $400 \mathrm{GeV}$, respectively. We also display the corresponding gluon-fusion results with the effective Higgs coupling approximation, and the weakboson-fusion ones within the SM. Again the gluon fusion and effective Higgs coupling results agree well for the whole $\Phi_{j j}$ region for the light Higgs boson case $\left(M_{h}=\right.$ $125 \mathrm{GeV})$ but not for the heavy one $\left(M_{h}=400 \mathrm{GeV}\right)$. One can observe the characteristic flat distribution of the weak boson fusion and the dip structures of the gluon-fusion results, respectively [7-12] for both the SM and SM4, which originate from the properties of the $C P$-even Higgs boson couplings to weak bosons and to gluons within the SM. The SM4 results are larger than the SM ones, and are close to the weak-boson-fusion results even in the central region $\Phi_{j j} \sim 0$, which will affect the Higgs boson search via the weak-boson-fusion channel significantly within the SM4.

In Tables I and II we present the cross sections for Higgs $+n$-jet $(n=0,1,2)$ production via gluon fusion at the $7 \mathrm{TeV}$ and $14 \mathrm{TeV}$ LHC in the SM and SM4 with the 



FIG. 3 (color online). Transverse momentum distribution of the harder jet for Higgs +2 -jet production via gluon fusion (GF) in the SM and the SM4 $\left(m_{b^{\prime}}=400 \mathrm{GeV}\right)$ at the LHC with $\sqrt{s}=$ $14 \mathrm{TeV}$ for $M_{h}=125 \mathrm{GeV}$ (left) and $400 \mathrm{GeV}$ (right). The minimal cuts of Eq. (3.1) are imposed. The corresponding effective Higgs coupling results (HEFT) are displayed as the blue dashed histograms.

minimal cuts of Eq. (3.1) and $m_{b^{\prime}}=300,400$ and $500 \mathrm{GeV}$, respectively. The NLO numbers for Higgs + 0-jet production have been obtained from HIGLU [40] by vetoing jets within the cuts of Eq. (3.1). ${ }^{1}$ We also exhibit the scale variation uncertainties by varying the renormalization and factorization scales $\mu_{R}=\mu_{F}$ from $M_{h} / 2$ to $2 M_{h}{ }^{2}$ With increasing $m_{b^{\prime}}$, the cross sections in the SM4 become smaller by $\leqslant 50 \%$ for $m_{b^{\prime}}$ varied from $300 \mathrm{GeV}$ to $500 \mathrm{GeV}$. The scale dependence of Higgs + 2-jet (1-jet) production is much larger than for Higgs +1 -jet $(0$-jet) production at $\mathrm{LO}$ due to the additional jet emission. The NLO numbers for Higgs +0 -jet production indicate

\footnotetext{
${ }^{1}$ It should be noted that the analysis of Ref. [41] used several approximations so that the full mass effects have not been taken into account consistently at NLO.

${ }^{2}$ Note that the scale dependences at LO are expected to underestimate the real theoretical uncertainties but serve as a rough estimate. Because of the large QCD corrections to Higgs + 1-jet and 2 jet production known in the SM in the heavy-top limit $[4,10]$, one expects that the full QCD corrections within the SM4 will be sizeable and positive in general.
}

FIG. 4 (color online). Azimuthal angle distribution between the two jets for Higgs +2 -jet production via gluon fusion (GF) in both the SM and SM4 $\left(m_{b^{\prime}}=400 \mathrm{GeV}\right)$, and via weak boson fusion (WBF) in the SM at the LHC with $\sqrt{s}=14 \mathrm{TeV}$ for $M_{h}=125 \mathrm{GeV}$ (left) and $400 \mathrm{GeV}$ (right). Both the minimal and weak-boson-fusion cuts of Eqs. (3.1) and (3.3) have been imposed. The corresponding effective Higgs coupling results (HEFT) are also shown.

moderate QCD corrections and a strong reduction of the residual scale dependence. We expect a similar strong reduction of the scale dependence for Higgs +1 -jet and 2-jet production at NLO after vetoing additional jets within the minimal cuts of Eq. (3.1). The strong uncertainty for Higgs + jet production at LO may hide the effects of extra heavy quarks partly in future LHC searches. However, due

TABLE I. Dependence of the Higgs $+n$-jet $(n=0,1,2)$ production cross sections (in $\mathrm{fb}$ ) via gluon fusion on the heavy quark masses and their scale uncertainty (by varying the factorization and renormalization scales by a factor of 2 up- and downwards) at the $7 \mathrm{TeV}$ LHC with the minimal cuts of Eq. (3.1) for $M_{h}=800 \mathrm{GeV}$.

\begin{tabular}{lcccc}
\hline \hline 7 TeV LHC & SM & SM4-300 & SM4-400 & SM4-500 \\
\hline $0 j$ LO & $17.4_{-4.4}^{+6.5}$ & $248_{-63}^{+92}$ & $150_{-38}^{+56}$ & $117_{-30}^{+44}$ \\
$0 j$ NLO & $27.7_{-2.8}^{+1.6}$ & $423_{-49}^{+36}$ & $254_{-29}^{+21}$ & $194_{-21}^{+14}$ \\
$1 j$ & $11.5_{-3.6}^{+5.8}$ & $170_{-53}^{+85}$ & $108_{-34}^{+54}$ & $86.7_{-26.9}^{+43.2}$ \\
$2 j$ & $3.97_{-1.5}^{+2.5}$ & $60.8_{-22}^{+39}$ & $40.7_{-15}^{+26}$ & $33.1_{-12}^{+21}$ \\
\hline \hline
\end{tabular}


TABLE II. Same as Table I, but for $14 \mathrm{TeV}$ LHC.

\begin{tabular}{lcccc}
\hline \hline 14 TeV LHC & SM & SM4-300 & SM4-400 & SM4-500 \\
\hline $0 j$ LO & $195.5_{-43.5}^{+60.8}$ & $2776_{-618}^{+864}$ & $1677_{-373}^{+522}$ & $1316_{-293}^{+409}$ \\
$0 j$ NLO & $227.2_{-11.4}^{+1.6}$ & $3506_{-239}^{+76}$ & $2107_{-142}^{+41}$ & $1597_{-96}^{+10}$ \\
$1 j$ & $154.2_{-44.1}^{+68.1}$ & $2273_{-651}^{+1003}$ & $1452_{-416}^{+641}$ & $1163_{-333}^{+514}$ \\
$2 j$ & $68.0_{-23.5}^{+39.8}$ & $1043_{-361}^{+611}$ & $705_{-244}^{+413}$ & $577_{-200}^{+338}$ \\
\hline \hline
\end{tabular}

to the large enhancement of the SM4 compared to the SM production rates and the accompanying jet information, the Higgs + jet production channels can still be quite useful for early discovery of a 4th generation within the SM context. Moreover, motivated by Refs. [42-45], we are presently performing the merging of the above calculated LO matrix elements of various jet multiplicities with parton showers in a consistent way, to reach fully exclusive description of events for Higgs production at the LHC in both the SM and SM4 [46].

For the experimental Higgs search within the SM4, the large modifications of the Higgs branching ratios may be highly relevant in addition. For light Higgs $\left(M_{h}<\right.$ $131 \mathrm{GeV}$ ), in analogy to the gluon-fusion processes, the decay width of the Higgs boson into gluons will be strongly enhanced, thus reducing all other branching ratios accordingly. Moreover, the additional heavy quark contributions will diminish the $W$-loop contribution to $H \rightarrow \gamma \gamma$, so that this rare decay mode will be suppressed even further. Because of these changes of the Higgs boson profile within the SM4 context, the search strategies have to be reinvestigated in detail. For heavier Higgs $\left(M_{h}>204 \mathrm{GeV}\right)$, the decay channels $H \rightarrow Z Z, W^{+} W^{-}$or $t \bar{t}$ remain the most dominate ones and the relevant branch ratios are nearly not changed, except near the threshold region $\left(2 m_{b^{\prime}, t^{\prime}}\right)$, where new decay channels of Higgs into 4th generation quarks are opened. Higgs boson searches via the weak-boson-fusion processes will be affected in a similar way by the modified Higgs branching ratios.

\section{SUMMARY}

We have presented the calculations of Higgs +1 -jet and 2-jet production processes induced by gluon fusion at the LHC, in both the SM and its fourth generation extention. We have compared the full results to the corresponding ones by using the effective Higgs couplings to gluons in the heavy quark approximation. As in Refs. [8,9], we have found that the approximation works well in both the SM and SM4, for light Higgs bosons and moderate transverse momenta of the jets, $P_{T} \lesssim m_{t}$, while otherwise the differences are large in general. Light Higgs boson production within the SM4 with jet(s) via gluon fusion is approximately enhanced by a factor 9 compared to the SM cross sections. For heavy Higgs bosons the production rates amount to more than 10 times the SM one and reach values of $\mathcal{O}(100 \mathrm{fb})$. However, these results are plagued by significant scale uncertainties, but they can still be useful for early discovery of the Higgs boson with extra heavy quark contributions. A fourth generation will affect the Higgs search strategy via the weak-boson-fusion channel strongly.

\section{ACKNOWLEDGMENTS}

This work is supported in part by the Concerted Research action Supersymmetric Models and their Signatures at the Large Hadron Collider of the Vrije Universiteit Brussels, by the IISN MadGraph convention 4.4511.10, by the National Natural Science Foundation of China, under Grants No. 10721063, No. 10975004, and No. 10635030, and the European Community's MarieCurie Research Training Network HEPTOOLS under contract MRTN-CT-2006-035505.
[1] H. M. Georgi, S. L. Glashow, M. E. Machacek, and D. V. Nanopoulos, Phys. Rev. Lett. 40, 692 (1978).

[2] S. Dawson, Nucl. Phys. B359, 283 (1991); A. Djouadi, M. Spira, and P. Zerwas, Phys. Lett. B 264, 440 (1991); D. Graudenz, M. Spira, and P. Zerwas, Phys. Rev. Lett. 70, 1372 (1993); M. Spira, A. Djouadi, D. Graudenz, and P. Zerwas, Nucl. Phys. B453, 17 (1995).

[3] R. V. Harlander and W. B. Kilgore, Phys. Rev. Lett. 88, 201801 (2002); C. Anastasiou and K. Melnikov, Nucl. Phys. B646, 220 (2002); V. Ravindran, J. Smith, and W. L. van Neerven, Nucl. Phys. B665, 325 (2003); M. Krämer, E. Laenen, and M. Spira, Nucl. Phys. B511, 523 (1998); S. Catani, D. de Florian, M. Grazzini, and P. Nason, J. High Energy Phys. 07 (2003) 028; S. Moch and A. Vogt, Phys.
Lett. B 631, 48 (2005); V. Ravindran, Nucl. Phys. B746, 58 (2006).

[4] D. de Florian, M. Grazzini, and Z. Kunszt, Phys. Rev. Lett. 82, 5209 (1999).

[5] B. Mellado, W. Quayle, and S. L. Wu, Phys. Rev. D 76, 093007 (2007); U. Langenegger, M. Spira, A. Starodumov et al., J. High Energy Phys. 06 (2006) 035; O. Brein and W. Hollik, arXiv:0710.4781.

[6] D. L. Rainwater and D. Zeppenfeld, J. High Energy Phys. 12 (1997) 005; D. L. Rainwater, D. Zeppenfeld, and K. Hagiwara, Phys. Rev. D 59, 014037 (1998).

[7] T. Plehn, D. L. Rainwater, and D. Zeppenfeld, Phys. Rev. Lett. 88, 051801 (2002).

[8] V. Del Duca, W. Kilgore, C. Oleari, C. Schmidt, and D. Zeppenfeld, Phys. Rev. Lett. 87, 122001 (2001). 
[9] V. Del Duca, W. Kilgore, C. Oleari, C. Schmidt, and D. Zeppenfeld, Nucl. Phys. B616, 367 (2001).

[10] John M. Campbell, R. Keith Ellis, and Giulia Zanderighi, J. High Energy Phys. 10 (2006) 028.

[11] V. Hankele, G. Klamke, and D. Zeppenfeld, arXiv:hep-ph/ 0605117; G. Klamke and D. Zeppenfeld, J. High Energy Phys. 04 (2007) 052.

[12] K. Hagiwara, Q. Li, and K. Mawatari, J. High Energy Phys. 07 (2009) 101.

[13] F. Campanario, M. Kubocz, and D. Zeppenfeld, arXiv:1011.3819.

[14] P. H. Frampton, P. Q. Hung, and M. Sher, Phys. Rep. 330, 263 (2000).

[15] H. J. He, N. Polonsky, and S.f. Su, Phys. Rev. D 64, 053004 (2001).

[16] G. D. Kribs, T. Plehn, M. Spannowsky, and T. M. P. Tait, Phys. Rev. D 76, 075016 (2007).

[17] B. Holdom, W. S. Hou, T. Hurth, M. L. Mangano, S. Sultansoy, and G. Unel, PMC Phys. A 3, 4 (2009).

[18] J. Erler and P. Langacker, Phys. Rev. Lett. 105, 031801 (2010).

[19] W. S. Hou, Chin. J. Phys. (Taipei) 47, 134 (2009).

[20] M. S. Carena, A. Megevand, M. Quiros, and C.E.M. Wagner, Nucl. Phys. B716, 319 (2005).

[21] Y. Kikukawa, M. Kohda, and J. Yasuda, Prog. Theor. Phys. 122, 401 (2009).

[22] LEP Electroweak Working Group, status of August 2009, http://lepewwg.web.cern.ch/LEPEWWG/.

[23] K. Nakamura et al. (Particle Data Group), J. Phys. G 37, 075021 (2010).

[24] T. Aaltonen et al. (CDF Collaboration), Phys. Rev. Lett. 104, 091801 (2010).

[25] W. J. Marciano, G. Valencia, and S. Willenbrock, Phys. Rev. D 40, 1725 (1989).

[26] T. Aaltonen et al. (CDF and D0 Collaborations), Phys. Rev. D 82, 011102 (2010).
[27] C. Anastasiou, S. Bucherer, and Z. Kunszt, J. High Energy Phys. 10 (2009) 068.

[28] C. Anastasiou, R. Boughezal, and E. Furlan, J. High Energy Phys. 06 (2010) 101.

[29] C. Anastasiou, R. Boughezal, and F. Petriello, J. High Energy Phys. 04 (2009) 003.

[30] J. Küblbeck, M. Böhm, and A. Denner, Comput. Phys. Commun. 60, 165 (1990); T. Hahn, Comput. Phys. Commun. 140, 418 (2001).

[31] T. Hahn and M. Perez-Victoria, Comput. Phys. Commun. 118, 153 (1999).

[32] A. Denner and S. Dittmaier, Nucl. Phys. B658, 175 (2003).

[33] G. J. van Oldenborgh and J. A. M. Vermaseren, Z. Phys. C 46, 425 (1990); G. J. van Oldenborgh, 66, 1 (1991).

[34] R. K. Ellis and G. Zanderighi, J. High Energy Phys. 02 (2008) 002.

[35] A. Denner and S. Dittmaier, Nucl. Phys. B734, 62 (2006).

[36] J. Ellis, M. K. Gaillard, and D. V. Nanopoulos, Nucl. Phys. B106, 292 (1976); M. A. Shifman, A. I. Vainshtein, M. B. Voloshin, and V.I. Zakharov, Yad. Fiz. 30, 1368 (1979) [Sov. J. Nucl. Phys. 30, 711 (1979)]; B. A. Kniehl and M. Spira, Z. Phys. C 69, 77 (1995).

[37] J. Alwall et al., J. High Energy Phys. 09 (2007) 028.

[38] H. Murayama, I. Watanabe, and K. Hagiwara, KEKReport No. 91-11, 1992.

[39] J. Pumplin, D. R. Stump, J. Huston, H. L. Lai, P. Nadolsky, and W. K. Tung, J. High Energy Phys. 07 (2002) 012.

[40] M. Spira, arXiv:hep-ph/9510347.

[41] E. Arik, O. Cakir, S. A. Cetin, and S. Sultansoy, Acta Phys. Pol. B 37, 2839 (2006).

[42] S. Catani, F. Krauss, R. Kuhn, and B. Webber, J. High Energy Phys. 11 (2001) 063.

[43] F. Krauss, J. High Energy Phys. 08 (2002) 015.

[44] S. Hoeche et al.,arXiv:hep-ph/0602031.

[45] J. Alwall et al., Eur. Phys. J. C 53, 473 (2007).

[46] J. Alwall, Q. Li, and F. Maltoni (to be published). 